\title{
Pediatric Appendicitis
}

\author{
Volkan Sarper Erikci* and Tunç Özdemir \\ Department of Pediatric Surgery, Sağlık Bilimleri University, Tepecik Training Hospital, Turkey
}

Received: December 13, 2017; Published: December 20, 2017

*Corresponding author: Volkan Sarper Erikci MD, Associate Professor of Pediatric Surgery, Kazim Dirik Mah. Mustafa Kemal Cad. Hakkıbey apt. No:45 D.10 35100 Bornova-İzmir, Turkey

\begin{abstract}
Appendicitis is the most common surgical diagnosis for children who present with abdominal pain to the emergency department. However, there are nonspecific examination findings and variable historical features during its presentation. Diagnosis of appendicitis in the pediatric patient may be challenging for the clinician dealing with these patients. It is important to have a high index of suspicion and taking a detailed history and physical examination. In diagnosis of appendicitis, adjunctive studies that may be useful are the white blood cell count, C-reactive protein, urinalysis, ultrasonography and computerized tomography when necessary. When appendicitis is suspected, patients should receive immediate surgical consultation, as well as volume replacement and antibiotics if indicated. With this timely approach it will be possible to prevent the significant morbidity that is associated with delayed diagnoses in younger patients.
\end{abstract}

\section{Introduction}

Acute appendicitis is the most common surgical emergency in children and adolescents. Although it is uncommon in preschool children, appendicitis may present at any age. The lifetime risk of developing appendicitis is $7 \%$ to $8 \%$, with a peak incidence in the teenage years [1]. There are 250,000 cases in the United States annually and the majority occur in children with the ages between 6-10 years. Nearly one-third of children with appendicitis have perforation at the time of surgical treatment. It affects males more frequently than females with male predominance (M:F ratio 3:2). There is a seasonal variation in the occurrence of appendicitis so that presentation of appendicitis is increased in the summer months with perforated appendicitis occurring more frequently in the fall and winter [2]. Despite advances and innovations in fluid resuscitation and antibiotics, appendicitis especially in preschool children is still associated with significant morbidity even mortality.

\section{Embryology and Anatomy}

As a continuation of the inferior tip of the cecum that becomes visible during the eighth week of gestation, appendix rotates to its final position which is the posteromedial aspect of cecum. According to Treves, there are several variations that can be classified, into four types: type 1 , the appendix is of fetal type with a funnel shape, type 2 , the appendix originates from the cecal fundus, type 3 , the appendix originates dorsomedially out of the cecum (most common type) and type 4 , the appendix originates directly beside the ileal orifice [3]. The position of the appendix varies among individuals. According to a study comprising 10,000 cases five positions can be identified [4]: ascending appendix in the retrocecal recess in $65 \%$ of cases (most common), descending appendix in the iliac fossa in $31 \%$ of cases, transverse appendix in the retrocecal recess in $2.5 \%$ of cases, paracecal and preileal ascending appendix in $1 \%$ of cases and paracecal and postileal ascending appendix in $0.5 \%$ of cases. During surgical treatment it is important to distinguish if the appendix is non-fixed (appendix libera) or fixed (appendix fixa)

With an average length of $8 \mathrm{~cm}$, the size of appendix varies from 0.3 to $33 \mathrm{~cm}$ and its diameter of ranges from 5 to $10 \mathrm{~mm}$. The blood comes form appendiceal branch of the ileocolic artery. There are a few submucosal lymph follicles present at birth and these increase to nearly 200 by the age of 12 years and the number of these lymph follicles decreases after the age of 30 .

\section{Etiology and Pathogenesis}

Obstruction of the appendiceal lumen is usually the first factor that starts the illness. If unresolved, this obstruction leads to vascular congestion, ischemic necrosis and subsequent infection. Inspissated fecal material or a fecalith are the most common causes that lead to appendiceal luminal obstruction. Other causes of obstruction include lymphoid follicle hyperplasia, foreign bodies, carcinoid or other tumors and rarely parasites. Fecaliths are found in approximately $40 \%$ of cases of acute appendicitis, $65 \%$ of cases of gangrenous appendicitis, and approximately $90 \%$ of cases of perforated appendicitis.

As the appendiceal mucosa continues to secrete the mucus after occlusion of the appendix lumen, this leads to a rapid increase of intraluminar pressure. Secretion of as little as $0.5 \mathrm{~mL}$ leads to an increase of pressure of approximately $45 \mathrm{~mm} \mathrm{Hg}$ according to the law of Laplace [5]. This phenomenon also explains the rapid 
perforation of appendix within a few hours of inflammation. For this reason all the patients with suspected acute appendicitis need hospitalization and close clinical monitoring if appendectomy is not to be performed immediately.

Distention stimulates nerve endings of visceral afferent pain fibers and leads to a dull, diffuse mid-abdominal pain that can not be easily located by the children. As the distention increases it causes reflex nausea and vomiting and once the inflammatory process has involved the serosal part of the appendix and parietal peritoneum, the characteristic finding of shift of the pain from periumbilical area to the right lower abdominal quadrant occurs. Impaired blood supply leads to compromise of the appendiceal mucosa allowing bacterial invasion of the deeper locations. Absorption of bacterial toxins and necrotic material causes fever, tachycardia, and leukocytosis. As the pressure increases, finally perforation occurs, usually through the infarcted areas. Although in some patients the disease may spontaneously resolve, untreated obstruction of the appendiceal lumen usually leads to gangrene and perforation.

\section{Clinical Presentation}

Although appendicitis can affect any age group, it is extremely rare in neonates and infants. Older children present clinical signs and symptoms which are quite variable in pattern and order of appearance. As a first symptom pain usually begins as a dull and vague pattern at the periumbilical area but with time it may localize to the right lower quadrant. Children usually report a gradual increase in pain intensity as the disease progresses. The anatomical variability in the locations of appendix vermiformis (i.e., retrocecal, pelvic, preileal) is common and may alter pain symptoms accordingly. Pelvic or retrocecal appendicitis may only present with right lower quadrant pain without periumbilical pain. Flank pain and referred testicular pain are also common symptoms in children with pelvic or retrocecal appendicitis. If the inflammed appendix has a close relationship with ureter or bladder, it may produce symptoms associated with urinary tractus such as urinary frequency, dysuria, urinary retention and bladder distention. It is traditionally known that severe gastrointestinal symptoms that develop prior to the onset of pain usually indicate a diagnosis other than acute appendicitis. On the other hand mild gastrointestinal symptoms such as decreased appetite, indigestion and changes in bowel habits may develop within a few hours of pain onset.

Typically patients with uncomplicated appendicitis have lowgrade fever. Fever above 38.6 degrees, tachycardia, and leukocytosis develop as a consequence of mediators released by ischemic tissues, white blood cells and bacteria. Children with appendicitis avoid movement and tend to lie in bed with their knees flexed. Hyperesthesia of the skin can be elicited by touching the skin of the patient with a stethoscope. Abdominal tenderness associated with appendicitis varies with the stage of the disease and location of the inflammed vermiform appendix. Classical "McBurney's point" that is the area one-third the distance from anterior superior iliac spine to the umbilicus, is the most common site of maximal tenderness that is found on the abdominal wall. Rectal tenderness may be observed in patients with pelvic appendicitis. In the case of malrotation the tenderness due to appendicitis may occur on unusual locations which may be away from the usual site.

There are some clinical signs that should produce high index of suspicion regarding appendicitis. These are namely, "Rovsings' sign" (palpation of left lower quadrant producing tendernes over the right iliac fossa) and it is a reliable indicator of appendicitis in childhood. The "psoas sign" that is commonly observed in retrocecal appendicitis and the "obturator sign" suggesting pelvic appendicitis are the other useful clinical signs that should prompt clinician to diagnose acute appendicitis early. The physical examination of a patient suspected to have appendicitis (especially pelvic appendicitis) is missing without rectal exam which may reveal a tender palpable mass or abscess.

Differential diagnosis of acute appendicitis has a wide spectrum of diseases and includes gastroenteritis, inflammatory bowel disease such as Crohn's disease, cholelithiasis, mesenteric adenitis, pancreatitis, peptic ulcer disease, Meckel's diverticulitis, constipation, intussusception and many other disease states (Table 1). Systemic diseases including diabetic ketoacidosis, lupus erythematosus, hemolytic uremic syndrome, sickle cell crisis, Henoch-Schönlein purpura, and parasitic infections may produce symptoms suggesting acute appendicitis. In females with certain situations such as ectopic pregnancy, ovarian torsion, developmental ovarian cysts and pelvic inflammatory disease a misdiagnosis of acute appendicitis may cause unnecessary surgical interventions. Pneumonia particularly affecting the right lower lobe, urinary tract diseases such as renal or ureteric stones, pyelonephritis, urinary tract infections can also mimic acute appendicitis. Children with cystic fibrosis have a higher incidence of acute appendicitis. A neonate with appendicitis should increase the suspicion of Hirschsprung's disease in the mind of the clinician.

Table 1: Differential diagnosis of pediatric appendicitis.

\begin{tabular}{|c|}
\hline Gastrointestinal Causes-Liver, Spleen and Biliary Tract Disorders \\
\hline Gastroenteritis \\
\hline Mesenteric lymphadenitis \\
\hline Constipation \\
\hline Trauma \\
\hline Peptic ulcer disease \\
\hline Meckel's diverticulum \\
\hline Inflammatory bowel disease \\
\hline Cholecystitis \\
\hline Intussusception \\
\hline Neoplasm (carcinoid, lymphoma) \\
\hline Food poisoning \\
\hline Intestinal obstruction \\
\hline Omental torsion \\
\hline Pancreatitis \\
\hline Volvulus \\
\hline Perforated viscus \\
\hline
\end{tabular}




\begin{tabular}{|c|}
\hline Hepatitis \\
\hline Cholecystitis \\
\hline Splenic infarction and splenic rupture \\
\hline Genitourinary causes \\
\hline Urinary tract infection \\
\hline Urinary calculi \\
\hline Pyelonephritis \\
\hline Pelvic inflammatory disease \\
\hline Ectopic pregnancy \\
\hline Ovarian/testicular torsion \\
\hline Hematocolpos \\
\hline Endometriosis \\
\hline Mittelschmerz \\
\hline Tubo-ovarian abscess \\
\hline Ovarian cyst rupture \\
\hline Metabolic disorders \\
\hline Diabetic ketoacidosis \\
\hline Hypoglisemia \\
\hline Porphyria \\
\hline Acute adrenal insufficiency \\
\hline Hematologic disorders \\
\hline Sickle cell disease \\
\hline Henoch-Schönlein purpura \\
\hline Hemolytic uremic syndrome \\
\hline Pulmonary causes \\
\hline Pneumonia (right lobe basilar) \\
\hline Pleuritis \\
\hline Pulmonary infarction \\
\hline Drugs and toxins \\
\hline Erythromicin \\
\hline Salicylates \\
\hline Lead poisoning \\
\hline Other causes \\
\hline Familial Mediterranean fever \\
\hline Infantile colic \\
\hline Parasitic infection \\
\hline Psoas abscess \\
\hline Functional pain \\
\hline Angioneurotic edema \\
\hline
\end{tabular}

\section{Diagnosis}

History and physical examination is important in diagnosing appendicitis in children. The most accurate diagnostic tool is perhaps the serial examinations by the same examiner while the child is cooperative with the clinician. Before starting the palpation of the abdomen, the child should be asked to point out the location of the abdominal pain. Cutaneous hyperesthesia is often an early finding derived from the T10 to L1 nerve roots. There is a mild abdominal pain that can not be localized at the early stages of the disease. As the disease progresses, localized tenderness is most often found at the McBurney's point. Rectal tendernes may be observed in pelvic appendicitis and tenderness midway between the twelfth rib and the posterior superior iliac spine is detected in patients with retrocecal appendicitis. If there is a malrotation acompanying the disease, position of the inflammed appendix has a role in the location of tenderness. Peritonitis ensues as the disease progresses to perforation with generalized abdominal rigidity. Rebound tenderness is seldom necessary for diagnosis and may cause unnecessary discomfort for children. Routine rectal examination in diagnosing appendicitis in childhood is a matter of debate and if other signs suggest to the diagnosis of appendicitis, rectal examination may be unnecessary. But it is especially helpful diagnostic tool for patients with pelvic appendicitis with abscess or those with uterine or adnexal pathologic conditions. It shonuld be re-emphasized that when the diagnosis is unclear, with the aid of serial physical examinations, it is possible to decrease the number of unnecessary surgical interventions that may increase risk to the patient if performed. This issue is very important because in a recent study, among the children undergoing appendectomy, $6.3 \%$ in Canada and $4.3 \%$ in the United States, are subsequently found to have a normal appendix and a misdiagnosis of appendicitis that leads to negative appendectomy ranges up to $30 \%$ [6].

The most aften used laboratory aids to diagnose appendicitis are white blood cell (WBC) count, absolute neutrophil count (ANC), and $\mathrm{C}$-reactive protein (CRP) but these tests alone are not helpful or predictive. Leukocyte count (WBC) above 10,000 is observed in greater than $90 \%$ of children with acute appendicitis. But normal leukocyte count may also be observed in $5 \%$ of patients with appendicitis. A shift to left is a usual finding and is of better diagnostic value compared to leukocyte count. A neutrophillymphocyte ratio of greater than 3.5 has a greater specifity and sensitivity in diagnosing appendicitis.

There are numerous appendicitis scoring systems that have been suggested as an adjunct in diagnosing appendicitis. Two systems namely the Alvarado score and the Pediatric Appendicitis Score (PAS) have been extensively studied but do not have $100 \%$ sensitivity and specificity in the diagnosis of appendicitis and they do not replace an experienced pediatric surgeon [7-9]. It has been documented that a pediatric surgeon can differentiate appendicitis from other abdominal disorders with $92 \%$ accuracy [10]. Urinalysis is helpful in differentiating urinary tract infections and urolithiasis from appendicitis. However when inflammed appendix is close proximity with ureter, hematuria and pyuria may also be detected even there is not urinary tract infection or urolithiasis.

Imaging studies may be useful in cases where the diagnosis is equivocal and plain film radiography may have a value. The incidence of presence of a fecalith that can be seen on direct roentgenograms ranges from $10 \%$ to $20 \%$ of cases with appendicitis and this ratio increases accordingly in patients with complicated appendicitis. Other subtle plain film findings are; sentinel loop in the right lower quadrant, lumbar scoliosis with a concavity to the right lower quadrant, mass effect due to pelvic abscess, loss of psoas shadow, obliteration of the properitoneal fat stripe. It is necessary to have a chest radiograph for ruling out right lower lobe pneumonia. 
Although it is usually useful tool in the management of children with intussusception, barium enema may rarely be performed in children suspected of having appendicitis. Barium enema findings in appendicitis include; incomplete filling of the appendix, extrinsic mass effect on the cecum or terminal ileum and irregularities of the appendiceal lumen.

With a sensitivity of $85 \%$ and specificity of greater than $90 \%$, ultrasonography (US) is an effective diagnostic tool if performed especially by skilled hands. Sonographic criteria for the diagnosis of appendicitis is demonstration of a noncompressible appendix that is $7 \mathrm{~mm}$ or larger in diameter, a wall thicker than $2 \mathrm{~mm}$, or an irregular wall that is rigid, and lacks peristalsis. Other findings that may be helpful in diagnosing appendicitis include absence of air in the appendiceal lumen, periappendiceal fat changes, visible appendicolith, complex mass, mesenteric lymph nodes and free fluid [11]. Advantages of US include lack of sedation, contrast agents and radiation during procedure [12]. On the other hand there are also disadvantages of US that include need for operator experience, a lack of regular availability during off hours, difficult visualization especially in obese children [13]. Computed tomography (CT) is also useful for inconclusive cases. It combines the advantages of many other imaging modalities, including rapid acquisition time and a lack of operator dependency [14].

The findings of an enlarged appendix ( $>6 \mathrm{~mm}$ ), appendiceal wall thickenning (>1 $\mathrm{mm}$ ) and appendiceal wall enhancement are useful diagnostic criteria that are found in CT. Comparing US and CT in diagnosing appendicitis in children, it has been proposed that US is more specific and CT is more sensitive. Magnetic resonance imaging (MRI) has a high diagnostic accuracy for appendicitis but it has certain disadvantages including limited utility, lack of availability in many centers, lengthy acquisition time, need for sedation or anesthesia and high cost compared to other imaging modalities [15]. It should be re-emphasized again that these radiological diagnostic modalities, if used routinely, cause hospital resource utilization and delay in surgical treatment. Besides potential cancer risk associated with ionizing radiation from CT should also be kept in mind and these imaging tools should be reserved for patients with uncertain findings related to appendicitis.

\section{Treatment}

There are several treatment modalities of appendicitis with a wide spectrum from nonoperative management to open or laparoscopic sugical interventions. Nonoperative management of appendicitis has been an interest for many scientists and several trials demonstrated successful nonoperative management of acute appendicitis in $70 \%$ to $85 \%$ of cases at the 1 -year follow-up $[16,17]$. On the other hand it has been stated in another meta-analysis that the combined failure and recurrence rates in nonoperative patients made this approach less effective overall [18]. Regarding the nonoperative management of pediatric appendicitis, children revealed a success rate ranging from $75 \%$ to $80 \%$ and in a recent study up to $89 \%$ success rate of nonoperative management has been reported $[19,20]$. It has also been demonstrated that compared to surgically treated patients, nonoperative patients reported higher quality-of-life scores at 30 days [20]. In some situations nonoperative management of appendicitis may be unseccessful and one of these predictors of failure of nonoperative management has been reported to be presence of appendicolith on imaging studies [21]. Another study on the nonoperative management of uncomplicated appendicitis demonstrated a failure rate of $60 \%$ and was halted early [22]. To sum up, nonoperative management for carefully selected children with acute appendicitis is possible and should be kept in mind.

In the surgical management of appendicitis, the goals are to minimize complications and cost, decrease patient anxiety, and improve quality of life. Although according to the traditional thinking that emergent appendectomy should be performed at the time of diagnosis, immediate surgical intervention is not considered mandatory for most patients. Many centers dealing with pediatric appendicitis now perform appendectomies in the day time for patients presenting at night time [23]. With this approach, the stress that occurs in the overnight appendectomies for both the children, their families and the surgeon is avoided.

Initial therapy starts with intravenous fluid resuscitation, broad spectrum antibiotic coverage and keeping the patient nothing per mouth. There is a trend toward decreasing the duration of antibiotic therapy. The recommended duration of antiobiotic therapy is preoperative plus 24 hours postoperative period for simple appendicitis and 10-day course of ampicillin, gentamicin, and metronidazole or clindamycin for complicated appendicitis and these therapies are known as gold standard. On the other hand bacteriologic epidemiology of appendicitis shows that both piperacillin/tazobactam and cefoxitin have been shown to be at least as effective as the triple-drug regimen, and may also decrase length of hospital stay and costs [24]. Nevertheless, total length of antibiotic therapy should be determined by the clinical condition of the patient (resolution of fever, pain, bowel function) and WBC count [25].

Complication and perforation rates are similar for patients undergoing surgery within 6 hours of admission compared to those undergoing surgery between 6-16 hours after admission to hospital. The majority of pediatric surgeons perform appendectomy within 8 hours after admission. Nevertheless after an expedient resuscitation, all the patients with appendicitis must undergo timely surgical exploration. Surgical treatment includes open technique and laparoscopic appendectomy. Except for specific situations including any surgical intervention having right lower quadrant incision such as Meckel's diverticulectomy or intussusception reduction, incidental appendectomy is no longer performed routinely and does not have any benefit. In traditional appendectomy that was first decribed by McBurney, a transverse or oblique right lower quadrant incision is performed and by splitting the muscles, abdominal cavity is entered and mesoappendix is divided followed by excision of the appendix at its base [26]. There are different techniques of management of appendiceal stump including simple ligation, ligation and inversion using a pursestring, or a pure inversion without ligature. The choice of stump management directly relates to attending surgeon. 
Since the first description of endoscopic appendectomy in 1983, laparoscopic appendectomies are being performed more commonly nowadays and today laparoscopic appendectomies have largely replaced the open surgery up to a percentage of 91\% [27]. There are several different operative approaches in the laparoscopic management of appendicitis including three-port laparoscopic intervention, transumbilical laparoscopic appendectomy, single port/insicion techniques. Advantages of laparoscopy in the treatment of pediatric appendicitis include shorter hospital stay, decreased postoperative pain and wound complications, ability to diagnose inconclusive cases, surgical ease in obese patients, and faster recovery after surgery. On the other hand there are disadvantages of laparoscopic approach in appendicitis which are a higher cost of equipments, longer operative time, time needed for learning curve in laparoscopy education, experience required for surgeons and increased incidence of intra-abdominal infection. It has been previously stated that complication rates are lower compared to open appendectomy except that the postoperative intra-abdominal abscess rate is higher with laparoscopic approach [28]. But this this topic seems to be changed as it has been found in the meta-analysis and multi-institutional reviews that there are no differences in intra-abdominal abscess rates following laparascopic surgery $[29,30]$. To sum up, laparoscopy is safe and effective means of performing an appendectomy in the treatment of pediatric appendicitis.

If a normal appendix is found at laparotomy (5-15\% of cases), the abdomen is systematically inspected for evidence of inflammatory bowel disease, a Meckel's diverticulum, mesenteric adenitis, peptic ulcer disease. In females fallopian tubes and ovaries should be identified and inspected for ovarian cysts or torsion, and for rare occurrence of isolated tubal torsion and pelvic inflammatory disease. Following open surgery or laparoscopic approach, there is a question of drain usage. Although it has long been stated that irrigating the abdominal cavity is not recommended in patients with simple appendicitis and may have a role in the management of complicated appendicitis, previous studies demonstrated that there was no data favoring irrigation for peritoneal contamination in perforated appendicitis [31]. Even an increase in abscesses resulting from the use of irrigation compared to no irrigation following laparoscopic surgical intervention has been reported [32].

Management of children with a palpable abdominal mass who present late (i.e., several days or weeks) is a controversial topic. Some suggest immediate appendectomy whereas others perform the procedure only a mass is confirmed either as a result of radiological diagnostic work up or during the surgery with the patient under anesthesia. In a meta-analysis evaluating early versus delayed appendectomy for perforated appendicitis concluded that delayed operation was associated with significantly less overall complications, wound infections, intra-abdominal abscesses, bowel obstructions, and reoperations [33]. On the other hand early appendectomy, compared to interval appendectomy, significantly reduced the time away from normal activities [33]. Opponents to interval appendectomy suggest that it is unnecessary because only $14 \%$ of patients have recurrent symptoms, and after initial diagnosis, recurrence is uncommon within 2 years.Nevertheless, it is very important that if an operation is to be performed, great care should be taken to avoid damage to adjacent structures such as small intestine, the fallopian tubes, ovaries and ureters. In the case of well localized periappendiceal abscess or phlegmon, after a prolonged antibiotic therapy (2-3 weeks) and CT or sonographic guided percutaneous abscess drainage may be an other option in the treatment of these children. The current standard for patients presenting with a palpable abdominal mass who are usually young children with perforation, is conservative management with interval appendectomy after 8 to 12 weeks.

\section{Complications}

Complication rates after appendectomy differ greatly with regard to the severity of the appendicitis. Complications are rarely seen after simple appendicitis, but are more oftenly seen in children with complicated appendicitis. Wound infection is the most common complication after appendectomy. With the worldwide usage of antibiotics, the rate of wound infection has fallen from $50 \%$ to less than $5 \%$, even in complicated appendicitis. Other complications of appendicitis include intra-abdominal abscess formation, wound dehiscence, postoperative intestinal obstruction, prolonged ileus, and rarely enterocutaneous fistula. It has been reported that the postoperative risk of an intra-abdominal abscess is approximately $20 \%$ for children with perforated appendicitis, and the risk for children with simple appendicitis to develop an abscess is less than $0.8 \%$ [31]. Tubal infertility and pylephlebitis may also be observed after surgical treatment for complicated appendicitis such as pelvic and subhepatic appendicitis, respectively. Sepsis and multisystem organ failure can ocur in young children with a prolonged illness before definite diagnosis. As the antibiotics have markedly decreased the incidence of infectious complications, the mortality rate for complicated apppendicitis has dropped dramatically to nearly zero. The overall morbidity in children with complicated appendicitis is less than $10 \%$.

\section{Conclusion}

Appendicitis occurs most commonly between the ages of 10 to 11 years. The classical signs and symptoms of migrating pain to the right lower quadrant, rebound tenderness are present in less than half of the children presenting with appendicitis. When the diagnosis is certain, the combination of evaluation and prompt surgical intervention is all that is needed. If the diagnosis of appendicitis is inconclusive, a period of observation including usage of scoring systems for evaluating the patient followed by radiological imaging modalities becomes a matter of necessity rather than of choice. As CT scans increase radiation exposure, US should be the choice of imaging modality in these patients. Laparoscopic approaches now constitute more than $90 \%$ of appendectomies in these patients even in cases with perforated appendicitis. In selected cases, appendicitis can be managed nonoperatively. Although provided that care is given by experienced clinicians and institutions, the best outcome for children with appendicitis may be anticipated, a small number of patients may still develop complications following 
surgical treatment of appendicitis. Nevertheless, the long-term outcome for the majority of children who undergo appendectomy is very good.

\section{References}

1. Addis DG, Shaffer N, Fowler BS, Fowler BS, Tauxe RV (1990) The epidemiology of appendicitis and appendectomy in the United States. Am J Epidemiol 132(5): 910-925.

2. Deng Y, Chang DC, Zhang Y, Webb J, Gabre-Kidan A, et al. (2010) Seasonal and day of the week variations of perforated appendicitis in US children. Pediatr Surg Int 26(7): 691-696.

3. Treves F (1885) Lectures on the anatomy of the intestinal canal and peritoneum in man. BMJ 1: 1(1262): 470-474.

4. Wakeley CP (1933) The Position of the vermiform appendix as ascertained by analysis of 10,000 cases. J Anat 67(pt 1): 277-283.

5. Arnbjornsson E, Bengmark S (1985) Obstruction of the appendix lumen in relation to pathogenesis of acute appendicitis. Acta Chir Scand 49(8): 789-791.

6. Cheong LH, Emil S (2014) Outcomes of pediatric appendicitis: an international comparison of the United States and Canada. JAMA Surg 149(1): 50-55.

7. Escriba A, Gamell AM, Fernandez Y, Quintillá JM, Cubells CL (2011) Prospective validation of two systems of classification for the diagnosis of acute appendicitis. Pediatr Emerg Care 27: 165-169.

8. Rezak A, Abbas H, Ajemian MS, Dudrick SJ, Kwasnik EM (2011) Decreased use of computed tomography with a modified scoring system in the diagnosis of pediatric acute appendicitis. Arch Surg 146: 64-67.

9. Goldman RD, Carter S, Stephens S (2008) Prospective validation of the Pediatric Appendicitis Score. J Pediatr 153: 278-282.

10. Williams RF, Blakely M, Fischer PE, Streck CJ, Dassinger MS, et al. (2009) Diagnosing ruptured appendicitis preoperatively in pediatric patients. J Am Coll Surg; 208(5): 819-825.

11. Schupp CJ, Klingmuller V, Strauch K, Bahr M, Zovko D, et al. (2010) Typical signs of acute appendicitis in ultrasonography mimicked by other disease? Pediatr Surg Int 26: 697-702.

12. Binkovitz LA, Unsdorfer KML, Thapa P, Kolbe AB, Hull NC, et al. (2015) Pediatric appendiceal ultrasound accuracy, determinacy and clinical outcomes. Pediatr Radiol 45(13): 1934-1944.

13. Yiğiter M, Kantarcı M, Yalçın O, Yalçin A, Salman AB (2010) Does obesity limit the sonographic diagnosis of appendicitis in children? J Clin Ultrasound 39(4): 187-190.

14. Bachur RG, Dayan PS, Bajaj L, Macias CG, Mittal MK, Stevenson, et al. (2012) The effect of abdominal pain duration on the accuracy of diagnostic imaging for pediatric appendicitis. Ann Emerg Med 60(5): 582-590.

15. Kulaylat AN, Moore MM, Engbrecht BW, Brian JM, Khaku A. et al. (2015) An implemented MRI program to eliminate radiation from the evaluation of pediatric appendicitis. J Pediatr Surg; 50(8): 1359-1366.

16. Di SS, Sibilio A, Giorgini E, Biscardi A, Villani S, et al. (2014) The NOTA Study (Non Operative Treatment for Acute Appendicitis): prospective study on the efficacy and safety of antibiotics (amoxicillin and clavulanic acid) for treating patients with right lower quadrant abdominal pain and long-term follow-up of conservatively treated suspected appendicitis. Ann Surg 260(1): 109-117.

17. Salminen P, Paajanen H, Rautio T, Nordström P, Aarnio M, et al. (2015) Antibiotic therapy vs appendectomy for treatment of uncomplicated acute appendicitis: the APPAC randomized clinical trial. JAMA 313(23): 2340-2348.

18. Mason RJ, Moazzez A, Sohn H (2012) Meta-analysis of randomized trials comparing antibiotic therapy with appendectomy for acute uncomplicated (no abscess or phlegmon) appendicitis. Surg Infect (Larchmt) 13(2): 74-84.

19. Hartwich J, Luks FI, Watson-Smith D (2016) Nonoperative treatment of acute appendicitis in children: a feasibility study. J Pediatr Surg 51(1): 111-116.

20. Minecci PC, Mahida JB, Lodwick DL, Sulkowski JP, Nacion KM, et al. (2016) Effectiveness of patient choice in nonoperative vs surgical management of pediatric uncomplicated acute appendicitis. JAMA Surg 151(5): 408-415.

21. Svensson JF, Patkova B, Almstrom M, Naji H, Hall NJ, et al. (2015) Nonoperative treatment with antibiotics versus surgery for acute nonperforated appendicitis in children. Ann Surg 261(1): 67-71.

22. Mahida JB, Lodwick DL, Nacion KM, Sulkowski JP, Leonhart KL, et al. (2016) High failure rate of nonoperative management of acute appendicitis with an appendicolith in children. J Pediatr Surg; 51(6): 903-1011.

23. Pepper VK, Stanfill AB, Pearl RH (2012) Diagnosis and management of pediatric appendicitis, intussusception, and Meckel diverticulum. Surg Clin North Am 92(3): 505-526.

24. Goldin AB, Sawin RS, Garrison MM, Zerr DM, Christakis DA (2007) Aminoglycoside-based triple-antibiotic therapy versus monotherapy for children with ruptured appendicitis. Pediatrics 119(5): 905-1011.

25. Lee SL, Islam S, Cassidy LD, Abdullah F, Arca MJ (2010) Antibiotics and appendicitis in the pediatric population: an American pediatric surgical association outcomes and clinical trials committee systematic review. J Pediatr Surg 45: 2181-2185.

26. McBurney CIV (1894) The incision made in the abdominal wall in cases of appendicitis, with a description of a new method of operating. Ann Surg 20: 38-43.

27. Jen HC, Shew SB (2010) Laparoscopic versus open appendectomy in children: outcomes comparison based on a statewide analysis. J Surg Res 161(1): 13-17.

28. Vegunta RK, Wallace LJ, Switzer DM, Pearl RH (2004) Laparoscopic appendectomy in children: technically feasible and safe in all stages of acute appendicitis. Am Surg 70: 198-201.

29. Jaschinski T, Mosch C, Eikermann M (2015) Laparoscopic versus open appendectomy in patients with suspected appendicitis: a systematic review of meta-analyses of randomised controlled trials. BMC Gastroenterol 15(1): 48.

30. Yau KK, Siu WT, Tang CN, Yang GP, Li MK (2007) Laparoscopic versus open appendectomy for complicated appendicitis. J Am Coll Surg 205(1): 60-65.

31. St Peter SD, Sharp SW, Holcomb GW, Ostlie DJ (2008) An evidencebased definition for perforated appendicitis derived from a prospective randomized trial. J Pediatr Surg 43(12): 2242-2245.

32. Hartwich JE, Carter RF, Wolfe L (2013) The effects of irrigation on outcomes in cases of perforated appendicitis in children. J Surg Res $180(2): 222-225$

33. Simillis C, Symeonides P, Shorthouse AJ, Tekkis PP (2010) A metaanalysis comparing conservative treatment versus acute appendectomy for complicated appendicitis (abscess or phlegmon). Surgery 147(6): 818-829. 


\section{(c) (P) This work is licensed under Creative Commons Attribution 4.0 License}

DOI: $10.32474 /$ PAPN.2017.01.000101

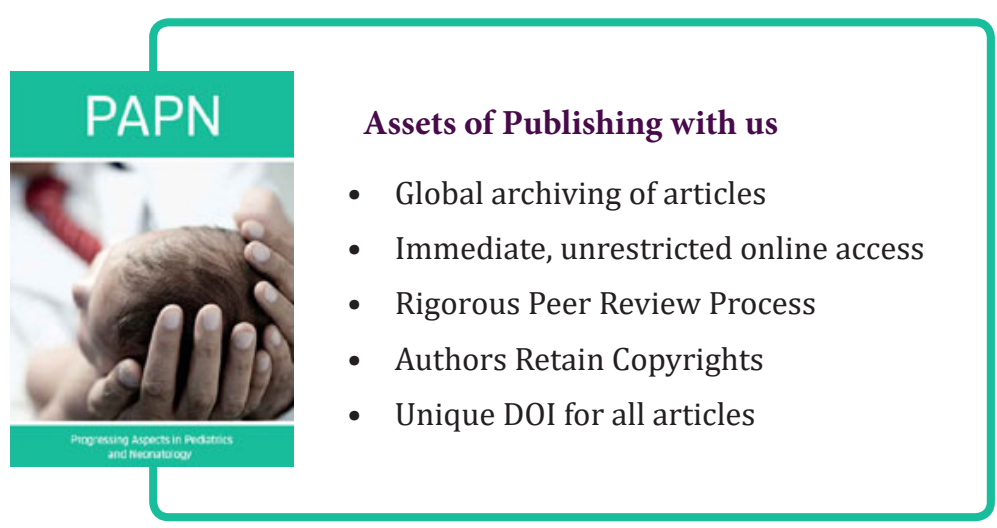

